\title{
SUBMILLIMETER-WAVE SIDEBAND GENERA'TION USING A PLANAR DIODE ARRAY
}

\author{
David S. Kurtz, Jeffrey L. Hesler, Jonathan B. Hacker*, \\ Thomas W. Crowe, David B. Rutledge**, Robert M. Weikle, II \\ School of Engineering and Applied Science \\ University of Virginia, Charlottesville, VA 22903
}

\author{
*Rockwell Science Center \\ 1049 Camino Dos Rios \\ Thousand Oaks, CA 91358
}

\section{ABSTRACT}

A 36 element array of planar Schottky diodes is used to mix the output of a $\mathrm{CO}_{2}$ pumped far infrared laser with a 1-20 GHz microwave source to generate tunable sidebands at 1.6 THz. The double sideband power was measured by heterodyne detection with a 1T23 corner cube Schottky diode for a calculated output power of $5.9 \mu \mathrm{W}$ with a 28 dB conversion loss.

\section{INTRODUCTION}

Over the past several years, instrumentation needs in the remote sensing and radio astronomy communities have steadily increased the demand for local oscillator sources operating at submillimeter wavelengths $[1,2]$. Tunable sources of submillimeter-wave radiation are rare. Far infrared lasers operate at discrete lines with no more than $200 \mathrm{MHz}$ of tunability, and the output of solid state sources drops off significantly as they approach $1 \mathrm{THz}$. Generation of tunable side bands using a whisker-contacted Schottky diode in a corner cube mount to mix a microwave source with a submillimeter laser has

This work was supported by the U.S. Army Research Office under grant DAAHO4-94-G-0112 and the U.S. Army National Ground Intelligence Center.

\author{
${ }^{* *}$ Division of Engineering and Applied Science \\ California Institute of Technology \\ Pasadena, CA 91125
}

been demonstrated to produce $10.5 \mu \mathrm{W}$ of output power at a drive frequency of $1.6 \mathrm{THz}$ [3]. Whisker-contacted Schottky diodes have been the forerunner for sensitive detectors but the recent trend has been to replace these devices with planar Schottky diodes [4]. Compared to their whisker-contacted counterparts, planar devices are rugged and more easily permit circuit topologies based on multi-diode configurations, but have not yet resulted in better performance [5]. A quasioptical array of planar Schottky diodes allows a convenient blend of optical and microwave technology appropriate for mixing submillimeterwave and microwave frequencies with greater power handling capabilities. In this paper, we report a $6 \times 6$ planar Sichottky diode array for generating sidebands using a $\mathrm{CO}_{2}$ pumped far infrared laser at $1.6 \mathrm{THz}$ and a $1-20 \mathrm{GHz}$ microwave source.

\section{DESIGN AND FABRICATION}

The sideband generator contains 36 planar Schottky diodes in a bowtie array placed on a quartz substrate. A drawing of the array is shown in figure 1. Microwave radiation is applied through the coplanar transition which is widened to accommodate an SMA connector. The laser power is incident normal to the array and a mirror is placed behind the array for impedance tuning. The array was monolith cally fabricated on GaAs 
Sideband Generator Grid with Microwave feed structure

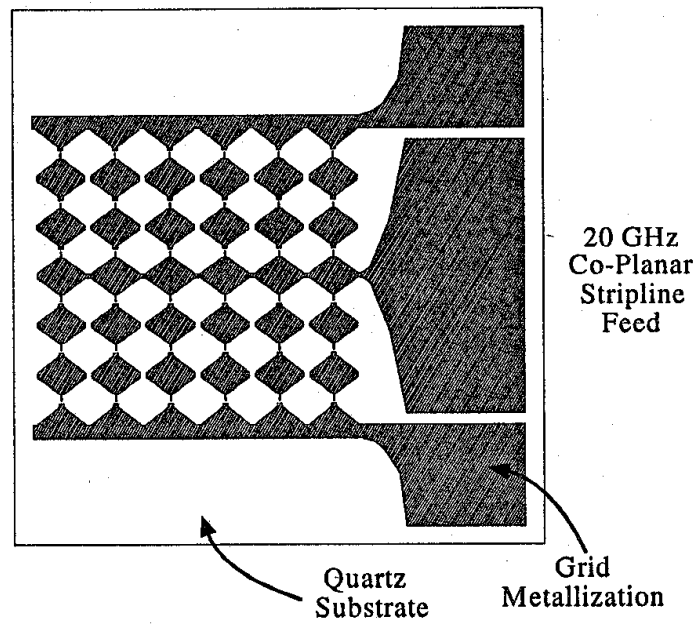

Figure 1: Bowtie array on quartz substrate with planar Schottky diodes at the feed point and microwave coplanar feed.

and a novel fabrication technique was used to remove the GaAs substrate and replace it with quartz [6]. The quartz provides a lower dielectric constant allowing a larger unit cell (70 $\mu \mathrm{m}$ compared to $30 \mu \mathrm{m}$ ) and has resulted in a wider bandwidth in simulations [7]. The array was designed to have large signal microwave frequencies modulate the impedance seen by the small signal laser power to produce small signal sidebands.

\section{MEASUREMENTS}

$\mathrm{A} \mathrm{CO}_{2}$ pumped far infrared laser operating at $1.6 \mathrm{THz}$ was mixed with a microwave source using the planar array of Schottky diodes. The sidebands were measured by heterodyne detection using a 1T23 whisker-contacted Schottky diode in a corner cube mount. Figure 3 shows a block diagram of the measurement setup. One laser was used to pump both the sideband generator and the detector. The Mach-Zehnder diplexer was used to couple half of the laser power to the sideband generator and half to the corner cube detector, while coupling all the generated sidebands to the

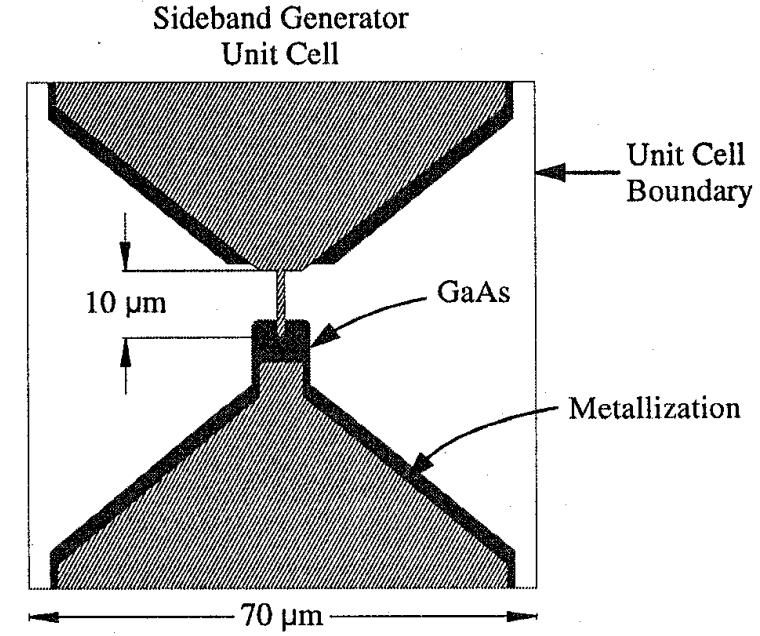

Figure 2: Unit cell of bowtie array showing finger contacting GaAs.

corner cube. The laser signal and sidebands were re-mixed in the corner cube detector reproducing the microwave frequencies which were then measured on a spectrum analyzer (HP 8565B). The laser path to the sideband generator could be blocked to determine the microwave power radiated directly from the signal generator to the corner cube detector. Because the sideband generator is an array of devices, the laser power is

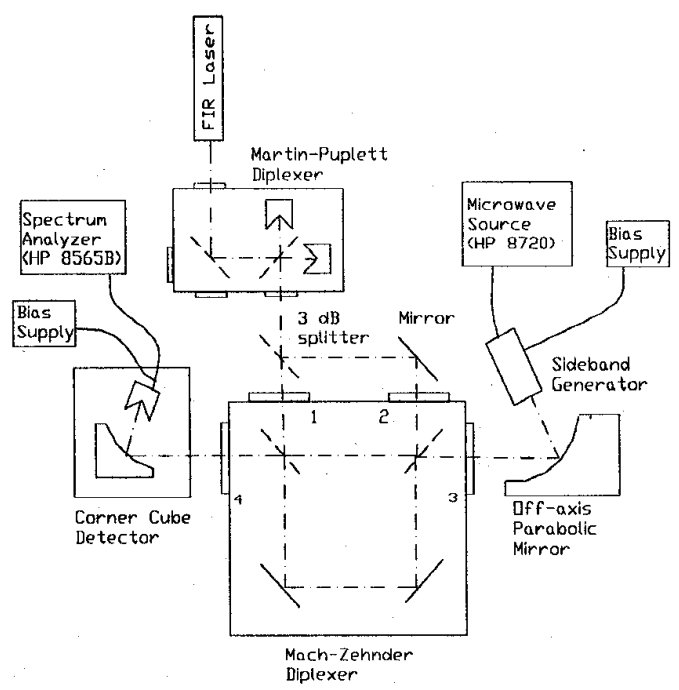

Figure 3: Block diagram of measurement system 
small signal for each individual device. The laser power, as a result does not significantly affect the microwave signal radiated directly from the array. Below $15 \mathrm{GHz}$ the radiated component, being as much as $30 \mathrm{~dB}$ below the sidebands, was an insignificant component of the total measured power. The estimated sideband power was calculated by correcting for the conversion loss of the corner cube detector which was determined from a hot/cold noise temperature measurement. The experiment was first performed using a 1T23 whisker-contacted Schottky diode as the sideband generator resulting in $7 \mu \mathrm{W}$ of double sideband power. (24 dB conversion loss). This is comparable to other results in the literature [3]. The planar array produced $5.9 \mu \mathrm{W}$ of double sideband power ( $28 \mathrm{~dB}$ conversion loss) at 1.8 $\mathrm{GHz}$. The tunability of the sidebands was demonstrated by changing the frequency of the microwave source as shown in figure 4 . The power was optimized at each frequency, so the frequency dependency is predominantly due to the Mach-Zehnder diplexer.

The microwaves modulate the laser by tuning the impedance of the array from a resistive load to an open circuit. The mirror acts as a back short and provides the impedance tuning necessary to present a matched load. The gaussian spreading of the laser beam from the

Frequency Dependency of Measurement System

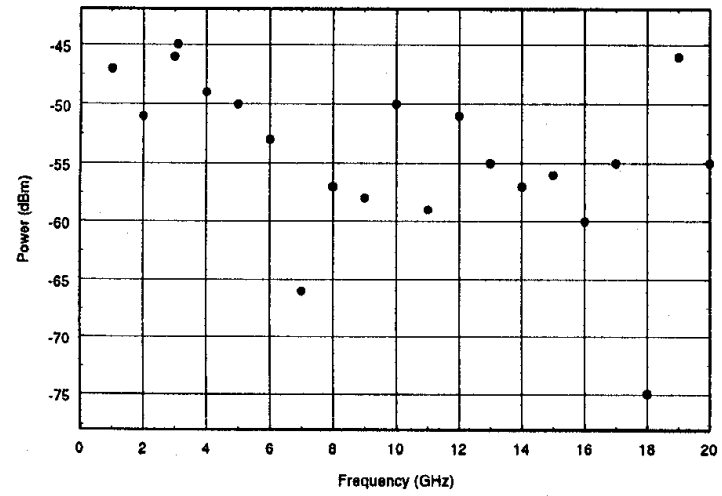

Figure 4: Sideband power measured by the spectrum analyzer as a function of frequency. The data at 19 and $20 \mathrm{GHz}$ are dominated by direct radiation.

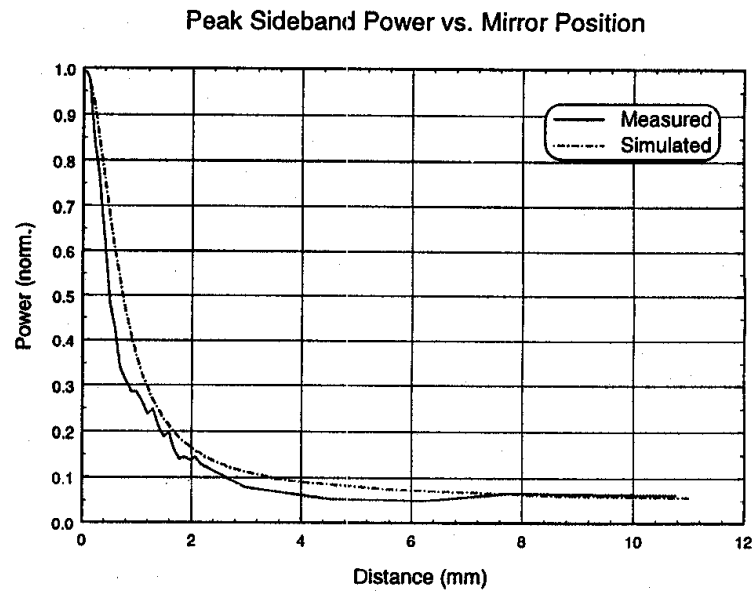

Figure 5: Just the peaks of the normalized sideband power as a function of the mirror position with a $1 \mathrm{~cm}$ radius crystalline quartz substrate lens facing the laser.

array to the mirror diminishes the mirror's effect. As the mirror is moved away from the array, the sideband power peaks and dips every $180^{\circ}$ (laser's half wavelength) with the maximums shrinking and the minimums growing. The peak power as a function of mirror position is shown in figure 5. In this measurement, the array is mounted on a $2 \mathrm{~cm}$ diameter quartz lens. The simulated curve was produced with HewlettPackard's Microwave and RF Design Systems (MDS) by using an ideal attenuator before the

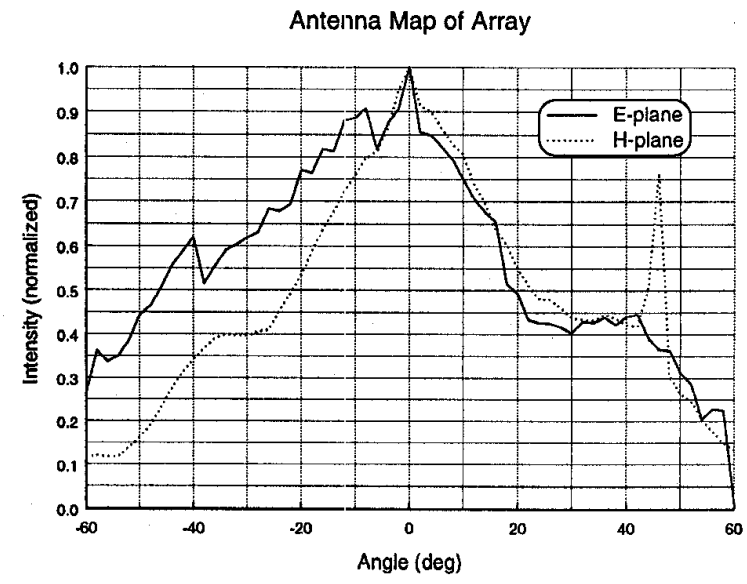

Figure 6: Video response as a function of angle. In this measurement, the array faces the laser and no substrate lens is used. 
mirror to model the gaussian coupling loss between the mirror and the array.

Antenna patterns were taken by placing the array in a gimble mount directly in front of the laser and measuring the video response as a function of angle as shown in figure 6 . The spike at $45^{\circ}$ is suspected to be radiation from the coplanar transition.

\section{SUMMARY AND FUTURE WORK}

In this work, we have reported a sideband generator array producing power levels close to those produced by whisker-contacted Schottky diodes in a corner cube mount. The array, biased at $2.8 \mathrm{~V}$ and $7 \mathrm{~mA}$, only has a $20 \mathrm{mV}$ video response to the laser power compared to several volts for the microwave signal. This demonstrates the large signal nature of the microwave source and the small signal nature of the laser power. The array is capable of handling much more input laser power than a single device sideband generator. The system was designed to produce sidebands in the reflected power analogous to the technique used with corner cubes. However, unlike corner cube sideband generators, power can transmit through the array. An array designed for transmissive sideband generation, analogous to a mechanical chopper, would simplify the system. Such an array could be placed at the output of the laser and directly modulate the laser beam without the need for beam splitters.

\section{REFERENCES}

[1] M.A. Frerking, "The Submillimeter Mission Heterodyne Instrument," Proceedings of the 2 nd Internat. Symposium on Space Terahertz Tech., pp. 17-31, Ann Arbor, MI, March 1991.

[2] J.W. Waters, "Submillimeter-Wavelength Heterodyne Spectroscopy and Remote Sensing of the Upper Atmosphere," Proceedings of the IEEE,
Special Issue on Terahertz Technology, vol. 80, no. 11, pp. 1679-1701, Nov. 1992.

[3] E.R. Mueller, J. Waldman, "Power and Spatial Mode Measurements of Sideband Generated, Spatially Filtered, Submillimeter Radiation," IEEE Trans. on Microwave Theory Tech., vol. 42, no. 10, pp. 1891-1895, Oct 1994.

[4] B.J. Rizzi, T.W. Crowe, N.R. Erickson, "A High-Power Millimeter-Wave Frequency Doubler using a Planar Diode Array," IEEE Microwave and Guided Wave Lett., vol. 3, no. 6, pp. 188-190, June 1993.

[5] J.L. Hesler, W.R. Hall, T.W. Crowe, R.M. Weikle, B. S. Deaver, R.F. Bradley, S.K. Pan, "Fixed-Tuned Submillimeter Wavelength Waveguide Mixers Using Planar Schottky-Barrier Diodes," IEEE Trans. on Microwave Theory Tech., vol. 45, no. 5, pp. 653-658, May 1997.

[6] W.L. Bishop, T.W. Crowe, R.J. Mattauch, H. Dossal, "Planar GaAs Diodes for THz Frequency Mixing Applications," $3^{\text {rd }}$ Int. Symp. Space Terahertz Technology, Ann Arbor, MI, pp. 600615, Mar. 1992.

[7] J.B. Hacker, "Grid Mixers and Power Grid Oscillators," Ph.D Thesis, California Institute of Technology. pp. 55, 1994. 